\title{
Activation of spinal neuregulin 1-ErbB2 signaling pathway in a rat model of cancer-induced bone pain
}

\author{
JINGJING JIANG ${ }^{1}$, JIN ZHANG $^{1}$, PENG YAO $^{2}$, XIUYING WU ${ }^{1}$ and KE LI ${ }^{1}$ \\ Departments of ${ }^{1}$ Anesthesiology and ${ }^{2}$ Pain Management, Shengjing Hospital \\ of China Medical University, Shenyang, Liaoning 110004, P.R. China \\ Received February 17, 2014; Accepted April 23, 2014
}

DOI: 10.3892/ijo.2014.2429

\begin{abstract}
Current therapies for cancer-induced bone pain (CIBP) are still limited. Until recently, the molecular mechanisms underlying the spinal cell-mediated CIBP remain largely unknown. To better understand the role of spinal cells in CIBP, we investigated the role of spinal neuregulin1 (NRG1)-ErbB2 signaling pathway in CIBP. In this study, a rat model of CIBP was established by intra-tibia inoculation of Walker 256 mammary gland carcinoma cells. Tibia bone destruction was evaluated using various methods of Walker 256 inoculation, radiobioassay and histological analysis. Pain-like behavior including thermal hyperalgesia and mechanical allodynia were determined by measuring paw withdrawal latency (PWL) and mechanical paw withdrawal thresholds (PWT). The expression level of mRNA or protein of target genes was examined by quantitative reverse transcription-polymerase chain reaction (qPCR) and western blot analysis, respectively. Bone destruction, as well as thermal hyperalgesia and mechanical allodynia, were observed at the 6th day following Walker 256 inoculation. A time-dependent increase in the levels of NRG1, ErbB2 and p-ErbB2 could be detected within three weeks after tumor cell injection. ErbB2 signaling inactivation by PD168393 treatment significantly attenuated pain-like behavior, associated with inhibition of Akt-1 and p38MAPK activation. Administration of exogenous NRG1 provoked pain-like behavior in rats by induction of activation of ErbB2, Akt-1 and p38MAPK, which could be blocked by ErbB2 inhibitor. Our results indicate that activation of NRG1-ErbB2 signaling pathway plays a critical role in the induction of CIBP. Akt-1 and p38MAPK may be potent players involved in the NRG1-ErbB2 pathway in CIBP.
\end{abstract}

Correspondence to: Dr Jin Zhang, Department of Anesthesiology, Shengjing Hospital of China Medical University, 36 Sanhao Street, Heping District, Shenyang, Liaoning 110004, P.R. China E-mail: zhangjin_sj@163.com

Key words: rat model, cancer-induced bone pain, neuregulin 1, ErbB2, Akt-1, p38 mitogen activated protein kinase

\section{Introduction}

Each year worldwide there are approximately nine million new cases of cancer reported, and 30-50\% of cancer patients suffer from different degrees of pain. Cancer-induced bone pain (CIBP) is a major clinical problem in patients with end-stage cancer, which affects the quality of life of cancer patients $(1,2)$. CIBP is usually induced by primary bone cancer or secondary bone metastasis from breast, prostate and lung cancer, among others. Although significant advances have been made in management of CIBP (3), it is still a difficult issue in clinical practice, because current treatments for this pain are limited due to inadequate knowledge of the mechanisms associated with CIBP. Therefore, further investigations on the mechanisms underlying cancer bone pain may lead to the development of new effective therapies.

In recent years, neuron-glia interaction has been considered a driving force for the development and maintenance of abnormal pain. Glial activation has been widely studied in different animal models, such as neuropathic pain after injuries to peripheral nerves (4) or the spinal cord (5), inflammatory pain after injection of inflammatory substances (e.g., formalin) into a hindlimb (6), cancer pain after inoculation of tumor cells (7), or orofacial pain after lesion of joint muscle (8). In these pain models, both microglia and astrocytes are activated, which interact with neurons and are involved in pain control. Activation of glial cells induces pro-inflammatory mediators, which trigger neural plasticity, leading to an increase of neuronal excitability and pain hypersensitivity. On the other hand, pain could be potentiated by growth factors such as brain-derived neurotrophic factor (BDNF), glia cell-derived neurotrophic factor (GDNF) and nerve growth factor (NGF); these are produced by activation of glial cells. The studies suggest that CIBP may be a mixture of inflammatory and neuropathic stimuli (9), and a variety of cellular signaling pathways are involved in this process, including the extracellular signal-regulated protein kinase (ERK)/mitogen activated protein kinase (MAPK) signaling pathway (10).

Neuregulin 1 (NRG1), a member of the NRG family of proteins, contains an epidermal growth factor (EGF)-like domain, which binds to the epidermal growth factor-like receptors ErbB3 and 4. These receptors are also found in microglia, and subsequently heterodimerize with ErbB2 (11). NRG1 has been implicated in the development of the 
central and peripheral nervous systems, cardiac tissue and tumors (12-14). The effects of NRG1 on cell proliferation, survival, migration and synaptic plasticity are associated with dimerization activation of ErbB2 (15-17). The NRG1-ErbB signaling pathway can trigger various intracellular signaling pathways including phosphatidylinositol 3-kinase (PI3K)/Akt and MAPK (18). Recent studies have revealed that following peripheral nerve injury, the level of NRG1 increased within the dorsal horn, leading to the activation of ErbB2, specifically within microglia and the pain-related hypersensitivity (19). However, whether the abnormal expression of NRG1-ErbB2 is involved in CIBP has not yet been reported.

We used a rat model of tibia bone cancer pain to investigate the effect of NRG1-ErbB2 receptor signaling in regulation of CIBP, providing insight into the novel mechanisms underlying CIBP regulation. Elucidating this mechanism may lead to the development of effective approaches for the management of bone cancer pain.

\section{Materials and methods}

Reagents. Dulbecco's modified Eagle's medium (DMEM) and fetal bovine serum (FBS) were obtained from Gibco, USA. PD168393 was purchased from Calbiochem, and HRG1 $\beta$ (ab50277) was obtained from Abcam, UK. Rabbit anti-ErbB2 (ab2428) and anti-p-ErbB2 (ab47755) were bought from Abcam. Mouse anti-NRG1 (SC-57384), rabbit anti-p-AKT-1 and rabbit anti-p-p38MAPK were purchased from Santa Cruz, USA. Anti-GAPDH was obtained from Sigma, USA. PVDF membrane and ECL Western Blotting Substrates were products of Millipore, USA and Pierce, USA, respectively. SYBR PrimeScript ${ }^{\mathrm{TM}}$ RT-PCR kit was obtained from Takara, Japan. Designed PCR primers were synthesized by Takara Biotechnology (Dalian) Co., Ltd, China.

Preparation of Walker 256 mammary gland carcinoma cells. The Walker 256 mammary gland carcinoma cell line was purchased from Cancer Institute and Hospital, Chinese Academy of Medical Sciences, Beijing, China. Cells were maintained in DMEM with $10 \%$ fetal bovine serum and penicillin/streptomycin $(100 \mathrm{U} / \mathrm{ml})$. The harvested cells were suspended at a concentration of $2 \times 10^{7}$ cells $/ \mathrm{ml}$. Female Sprague-Dawley (SD) rats, weighing 80-100 g, were provided by Experimental Animal Center, Shengjing Hospital of China Medical University, Shenyang, China. Each rat received intra-abdominal injection of $0.5 \mathrm{ml}$ of the cell suspension. Ascites formed 6-7 days following tumor cell injection. Thereafter, 5 to $10 \mathrm{ml}$ of ascites were collected and cell were harvested by centrifugation, then suspended in phosphate buffer saline (PBS) at a density of $1 \times 10^{7}$ cells $/ \mathrm{ml}$. Same amount of cells were taken as a negative control by heat inactive treatment in a $100^{\circ} \mathrm{C}$ water bath for $10 \mathrm{~min}$.

Establishment of rat model of cancer-induced bone pain. Female SD rats as mentioned above, weighing 200-220 g, were routinely housed in light (12 h dark/12 h light) and temperature-controlled $\left(22-24^{\circ} \mathrm{C}\right)$ rooms and had free access to food and water. Experimental animal use was approved by the Experimental Ethics Committee of Shengjing Hospital of China Medical University. Animal experiments were carried out in accordance with the Guidelines laid down by the NIH in the US regarding the care and use of animals for experimental procedures. The rat model of CIBP was established as previously described (20). In brief, rats were anesthetized by intraperitoneal injection of $10 \%$ chloral hydrate $(300 \mathrm{mg} / \mathrm{kg})$. The right leg was shaved and the skin was disinfected with $70 \%(\mathrm{v} / \mathrm{v})$ ethanol. A $1-\mathrm{cm}$ incision was made in the skin, and the tibia was carefully exposed with minimal damage to blood vessels and nerve. The tibia was pierced using a 23-gauge needle. A total of $10 \mu \mathrm{l}$ of cell suspension in PBS that contains 0.1 million $\left(10^{5}\right)$ Walker 256 mammary gland carcinoma cells were slowly injected into the right tibia cavity of each rat using a $25-\mu 1$ microinjection syringe within 2 min. The injection site was sealed with bone wax, followed by irrigation with sterile normal saline.

Intrathecal catheterization. Intrathecal catheterization was carried out as previously described (21). In brief, animals were anesthetized by $10 \%$ chloral hydrate $(300 \mathrm{mg} / \mathrm{kg}$, i.p.). A PE10 intrathecal catheter was inserted $2 \mathrm{~mm}$ cephalad into the rat lumbar subarachnoid space at the level of L3-L4 intervertebrae. The tip of the catheter was located near the lumbar enlargement of the spinal cord for intrathecal administration of the drugs. The catheter was tunneled subcutaneously and externalized through the skin in the neck region. Two days later, $20 \mu \mathrm{l}$ lidocaine $(20 \mathrm{~g} / \mathrm{l})$ was injected intrathecally to rats without impaired movement. If these animals showed lower limb paralysis within $30 \mathrm{sec}$ of injection time, it was a successful sign for catheterization.

Experimental design. To verify the effect of ErbB2 signaling on the regulation of CIBP, PD168393, a structurally related irreversible-binding ErbB2 inhibitor (22), was used. Animals were randomly divided into four groups, including: sham operation ( $n=6$ for each time point), CIBP $(n=6$ for each time point), CIBP + PD168393 treatment $(n=12)$, and CIBP + vehicle treatment $(n=12)$. In CIBP group, intact Walker 256 mammary gland carcinoma cells were injected into the tibia cavity of rat without any treatment, whereas in the sham operation group, the same amount of heat-inactivated cells (negative control) were injected. In the CIBP + PD168393 or CIBP + vehicle groups, $10 \mu \mathrm{g}$ of PD168393 was dissolved in $5 \%$ DMSO $(10 \mu \mathrm{l})$, or the same volume of DMSO was administered by intrathecal injection on the 6th day after pain model establishment, respectively. Drug was injected daily for 9 successive days.

To observe the effect of NRG1 on the induction of cancer-induced bone pain, animals were randomly divided into three groups, sham operation, HRG1 $\beta$ and HRG1 $\beta$ + PD168393. Six animals were used for each time point. In the sham operation group, $10 \mu \mathrm{l}$ of normal saline was administered by intrathecal injection; while in the HRG1 $\beta$ group, $10 \mu \mathrm{l}$ of exogenous NRG1 (HRG1 $\beta, 0.4 \mathrm{ng} / \mu \mathrm{l}$ ) was administered by intrathecal injection. In the HRG1 $\beta+$ PD168393 group, $1 \mathrm{~h}$ post-injection of $10 \mu \mathrm{g}$ PD168393, the same dose of HRG1 $\beta$ was injected. Drug injection was performed once a day for three successive days.

Behavior tests. Thermal hyperalgesia was determined by measuring paw withdrawal latency (PWL) during heat stimu- 
Table I. Specific primers for qRT-PCR.

Annealing

Target gene

Primer sequences

temperature $\left({ }^{\circ} \mathrm{C}\right)$

NRG-1

Forward: 5'-GGCAGTCAGCCCCTTTGTG-3'

58

Reverse: 5'-TGCAGGGTTGTGATGAAAGGA-3'

ERBB2

Forward: 5'-CCTGCCTCCACTTCAATCAT-3'

59

Reverse: 5'-CAGGATCCCACTTCCGTAGA-3'

Akt-1

Forward: 5'-CTCTGCATTGCCGAGTCC-3'

58

p38MAPK

Reverse: 5'-GGTTCCTCCCCAGCACAT-3'

58

Reverse: 5'-CACAGTGAAGTGGGATGGA-3'

GAPDH

Forward: 5'-ATGACTCTACCCACGGCAAG-3'

60

Reverse: 5'-GGAAGATGGTGATGGGTTTC-3'

lation, using a thermal stimulator manufactured by Biomedical Engineering Institute of Chinese Academy of Medical Science (BME-410A). As described previously $(23,24)$, rats were placed in a container $(22 \mathrm{~cm} \times 12 \mathrm{~cm} \times 12 \mathrm{~cm}$; length $\mathrm{x}$ width $\mathrm{x}$ height) with a smooth glass floor. All rats were given 30 min to acclimate to the testing environment. A heat source was focused on a portion of the hindpaw, which was flush against the glass and a radiant thermal stimulus was delivered to that site. The stimulus shut off automatically when the hindpaw moved, or after $20 \mathrm{sec}$ to avoid tissue damage. Thermal stimuli were delivered three times to each hindpaw at 10 -min intervals. Baseline thermal withdrawal latency for each rat was measured one day prior to experiment.

Mechanical paw withdrawal thresholds (PWT) were measured with von Frey filaments using a modification of the up-and-down method (25). Rats were placed in transparency organic glass cages with wire mesh bases. A total of $30 \mathrm{~min}$ of time for the acclimation of testing environment was given. A series of von Frey filaments with bending pressures ranging from 0.6 to $26 \mathrm{~g}$ were used. Those von Frey hairs were pressed perpendicularly against the plantar surface of the hind paw and held for 3-4 sec. Each von Frey filament was applied 5 times with 15 -sec intervals. A positive response was regarded in presence of sharp withdrawal of the paw, licking of the paw or flinching upon removal of the von Frey filament. Baseline PWT for each rat was measured one day prior to experiment.

Radiobioassay analysis. To examine tibial destruction from the inoculated tumor, animals were radiographed at 6,14 and 21 days following tumor cell inoculation. Animal right hind limbs were placed on X-ray film and exposed to an X-ray source for $1 / 20 \mathrm{sec}$ at $40 \mathrm{kV}$ (p) at day 12 post-inoculation. The radiographic images were visually quantified using the scale developed by Schwei et al (26).

Histological examination. Animals were euthanized under anesthesia three weeks following establishment of the CIBP model. The right tibia and surrounding tissues were carefully removed, fixed in $4 \%$ paraformaldehyde (PFA), decalcified in
PFA solution containing 5\% formic acid, embedded in paraffin and sectioned. Sections were deparaffinized by immersing the tissues in dimethylbenzene, followed by rehydration. Haematoxylin and eosin (H\&E) staining was performed according to standard procedures.

Real-time quantitative reverse transcription-polymerase chain reaction ( $q R T-P C R)$. Animals were sacrificed and the L4-L6 lumbar spinal cord segments were dissected. Total RNA was extracted using TRIzol Reagent according to the manufacturer's protocol (Invitrogen, USA). cDNA was then transcribed using PrimeScript ${ }^{\mathrm{TM}}$ RT Enzyme Mix I, Oligo (dT) primer, Random 6 mers and 5xPrimeScript ${ }^{\mathrm{TM}}$ Buffer (Takara). The sequences of specific primers are listed in Table I. PCR reactions with $25 \mu 1$ volumes was carried out using a LightCycler Quantitative PCR instrument (Roche, USA) in the following conditions: a preheating cycle at $95^{\circ} \mathrm{C}$ for $30 \mathrm{sec}$, followed by 40 cycles at $95^{\circ} \mathrm{C}$ for $5 \mathrm{sec}$, specific annealing temperatures are indicated in Table I for $15 \mathrm{sec}$, and $72^{\circ} \mathrm{C}$ for $10 \mathrm{sec}$. The relative expression from amplified RNA samples was calculated using the $2^{-\Delta \Delta \mathrm{CT}}$ method (27).

Western blot analysis. Protein was extracted from L4-L6 lumbar spinal cord segments by homogenization with radio immunoprecipitation assay (RIPA) lysis buffer and protein concentration was measured using a bicinchoninic acid (BCA) Protein Assay reagent kit (Santa Cruz) according to the manufacturer's instructions. Equal amounts of protein extracts were then separated by 6 or $10 \%$ sodium dodecyl sulfate-polyacrylamide gel electrophoresis (SDS-PAGE), transferred to a PVDF membrane, then blocked with $5 \%(\mathrm{w} / \mathrm{v})$ non-fat dry milk in tris buffered saline plus Tween-20 (TBS-T; 0.1\% Tween-20), followed by overnight incubation with primary antibodies at $4^{\circ} \mathrm{C}$. The applied primary antibodies were diluted with TBS-T as follows: anti-ErB2 (1:200), anti-p-ErB2 (1:200), anti-NRG1 (1:200), anti-p-Akt-1 (1:500), anti-p-p38MAPK (1:500), and anti-GAPDH $(1: 1,000)$. After washing with TBS-T, the membranes were incubated with HRP labeled secondary antibodies $(1: 5,000)$. The expression level of target proteins was determined by analysis using an Image Acquisition 
A

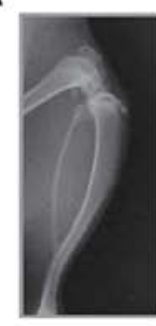

Normal

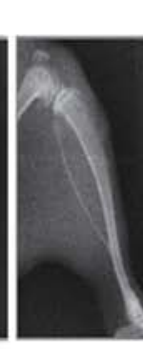

sham

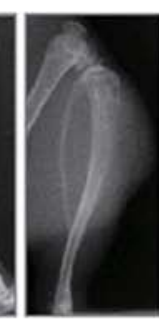

$6 \mathrm{~d}$

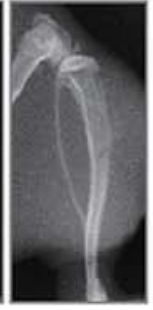

$14 \mathrm{~d}$

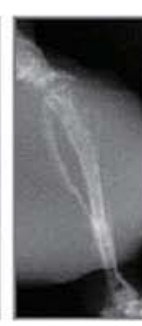

$21 \mathrm{~d}$
CIBP

B

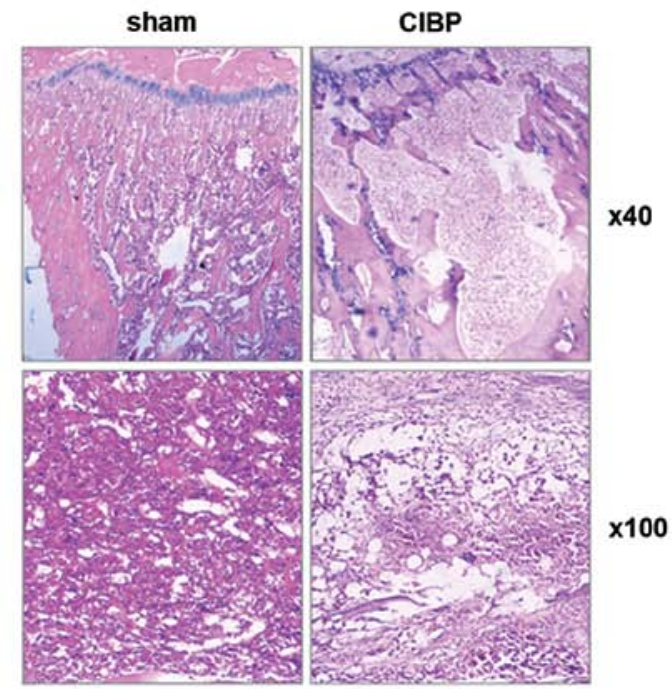

C

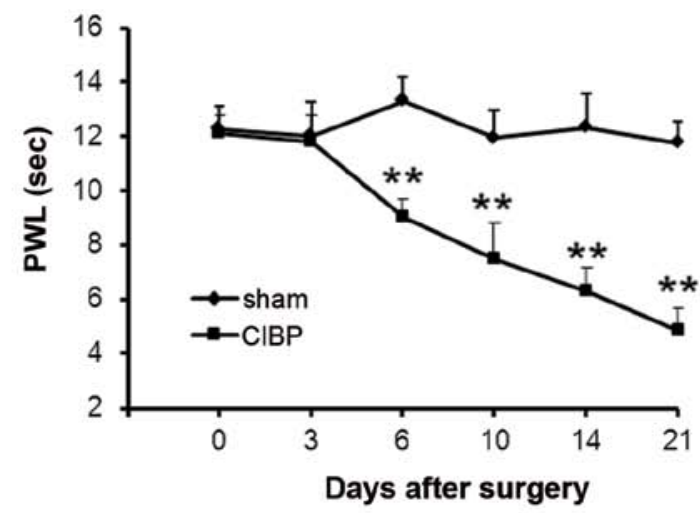

D

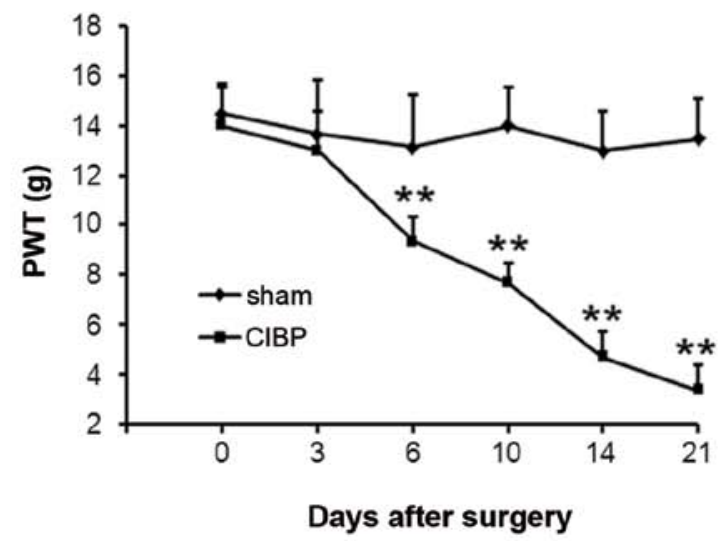

Figure 1. Tibia bone destruction and changes of pain behavior by Walker 256 inoculation. (A) Radiographs of the tibial bone inoculated with heat-inactivated (sham) or intact Walker 256 mammary gland carcinoma cells (CIBP) at the 6th, 14th and 21st day after inoculation. Healthy rats were used as control (normal). Representative images are shown. (B) Histology of tibial bone destruction stained by hematoxylin and eosin at 21 st day after Walker 256 inoculation. Magnification, $\mathrm{x} 40$ or $\mathrm{x} 100$. (C) Thermal hyperalgesia determined by measuring paw withdrawal latency (PWL) during heat stimulation using a thermal stimulator on indicated day after inoculation. (D) Paw withdrawal threshold (PWT) to a static mechanical stimulus assessed using von Frey filaments and the up-and-down method. ${ }^{* *} \mathrm{P}<0.01$ compared with sham; $\mathrm{n}=6$.

and Analysis System (GDS8000, UVP, Inc., USA). The densitometric values were semiquantified by Bio-Rad and Quantity One imaging analysis software. The housekeeping protein, GAPDH, was used as an internal control.

Statistical analysis. Statistical analysis was performed using SPSS 16.0 software. Data were presented as the mean \pm standard deviation (SD). P-value $<0.05$ was considered to be significant. Data were analyzed using a one-way and repeated measures analysis of variance (ANOVA) followed by Bonferroni-corrected post-hoc tests.

\section{Results}

Time course of pain development in the rat model of CIBP. Radiological and histological examinations were used to evaluate bone destruction in the rat model of CIBP. As shown by X-ray images, no obvious alternation of tibia structure was found at either the 6th, 14th, or 21st day after inoculation of heat-inactivated Walker 256 mammary gland carcinoma cells (sham) (Fig. 1A; bone destruction score: $0 ; n=6$ ). However, 6 days following inoculation of Walker 256 tumor cells, defects within the proximal tibial epiphysis were detected. These defects became more prominent at the 14th day (bone destruction score: day $6,1.66 \pm 0.52$; day $14,2.66 \pm 0.54$; $\mathrm{n}=6$ for each). Further deterioration was found at 21 days post-injection, with the occurrence of cortical destruction, tumor dissemination and soft tissue tumors (bone destruction score: $4.33 \pm 0.43 ; n=6$ ). Histological examination showed that bone marrow spaces were infiltrated with tumor cells at the 21st day following tumor cell inoculation (Fig. 1B). Moreover, bone trabecula defects, loss of normal bone structure, cortical destruction, and soft tissue tumors were observed in the CIBP model group. Bone destruction was not observed in animals within the sham operation group. A progressive decrease in both PWL and PWT, corresponding to thermal and mechanical stimulation of the inoculated hind paw, occurred at the 6th day after tumor cell injection (Fig. 1C and D). Compared with the sham operation group, significant differences in PWT and PWL values in the CIBP group were found at days 6,10 , 14 and 21 following tumor cell inoculation $(\mathrm{P}<0.01)$.

Sustained activation of NRG1 and ErbB2 signaling pathway in the spinal dorsal tissues of the rat CIBP model. Next, 
A

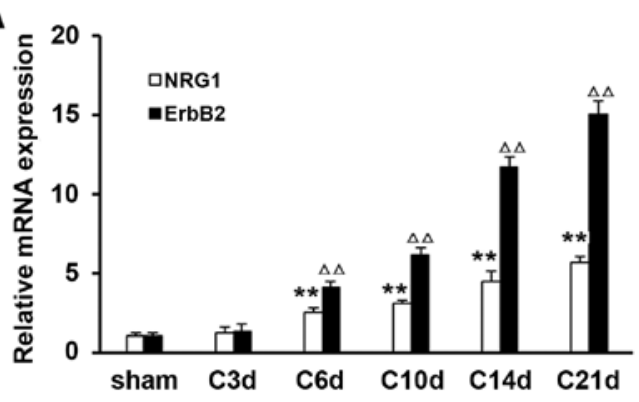

B
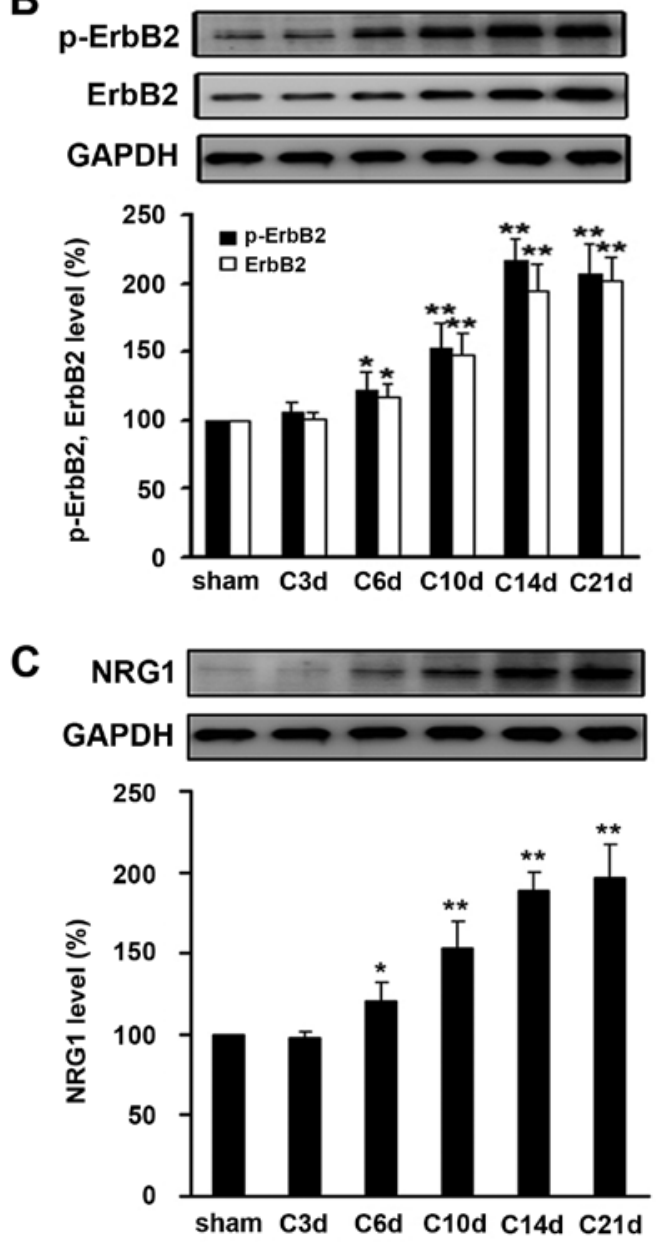

Figure 2. Sustained activation of NRG1 and ErbB2 in the spinal dorsal tissues after intra-tibia inoculation with Walker 256 mammary gland carcinoma cells. (A) As indicated day after Walker 256 inoculation (day 3, $6,10,14$ and 21), the mRNA levels of ErbB2 and NRG1 were determined by real-time qRT-PCR. Relative mRNA expression (target gene/GAPDH) was quantified from six animal samples. NRG1 vs. sham, ${ }^{*} \mathrm{P}<0.05,{ }^{* *} \mathrm{P}<0.01$; ErbB2 vs. sham, ${ }^{\triangle} \mathrm{P}<0.05,{ }^{\triangle} \mathrm{P}<0.01$. (B) Protein expressions of ErbB2, (B) p-ErbB2 and (C) NRG1 were examined by western blot analysis. Relative protein expression (target protein/GAPDH) was quantified from six animal samples. " $\mathrm{P}<0.05,{ }^{* *} \mathrm{P}<0.01$ compared with sham (day 0 ); $\mathrm{n}=6$.

we examined the expression level of NRG1 and ErbB2 in the tissues of L4-L6 spinal cord space from rats inoculated with intact or heat-inactivated Walker 256 mammary gland carcinoma cells. Compared with sham operation control, the expression levels of mRNA and protein of NRG1, ErbB2 and

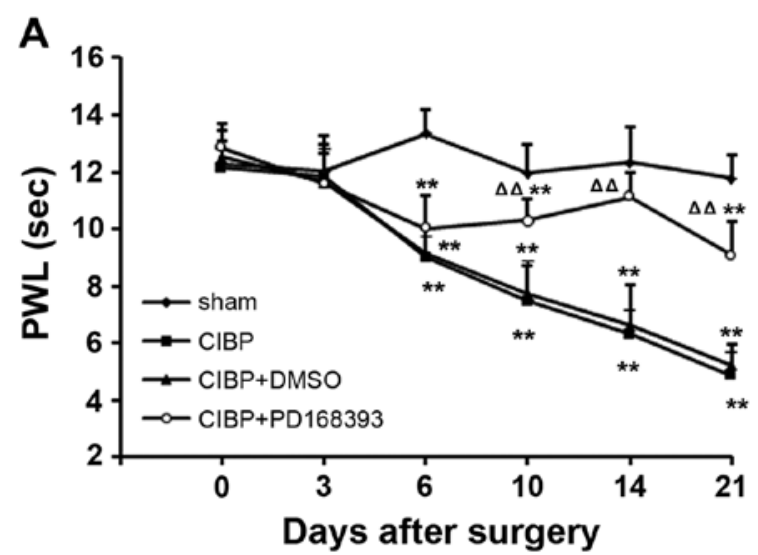

B

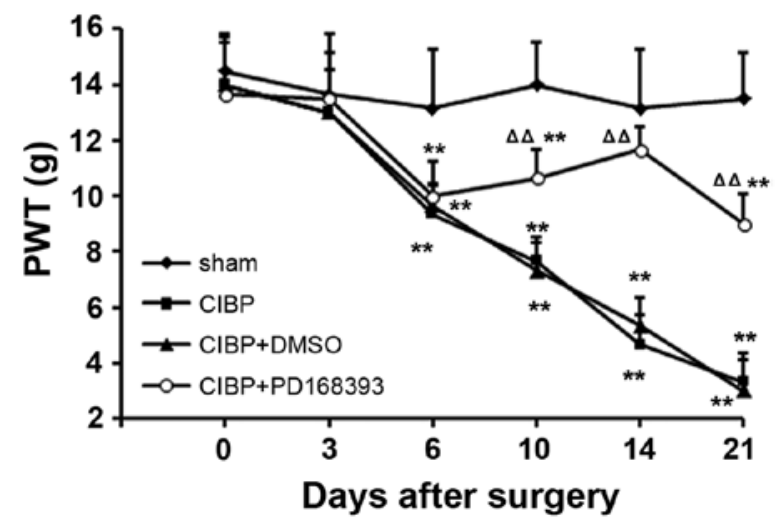

Figure 3. Inhibition of ErbB2 signaling attenuates pain-like behavior in tumor-inoculated rats. A total of $10 \mu \mathrm{g}$ of PD168393 dissolved in $5 \%$ DMSO $(10 \mu 1)$ or equal volume of DMSO was injected into the tibia cavity at day 6 after Walker 256 inoculation, in either CIBP+PD168393 or CIBP+DMSO group. Drug was injected daily for 9 consecutive days. (A) PWL and (B) PWT values were assessed on the indicated day after Walker 256 inoculation as previously described. Data were calculated from six animals. ${ }^{*} \mathrm{P}<0.05,{ }^{* *} \mathrm{P}<0.01$ compared with sham; ${ }^{\Delta} \mathrm{P}<0.05,{ }^{\Delta} \mathrm{P}<0.01$ compared with CIBP group; $n=6$.

p-ErbB2 were significantly elevated at day 6 after tumor cell inoculation $(\mathrm{P}<0.05)$ (Fig. 2). Time-dependent upregulation of NRG1, ErbB2 and p-ErbB2 expression was observed within three weeks following tumor cell injection. These observations suggest that sustained activation of NRG1 and ErbB2 signaling in the spinal dorsal tissues after intra-tibia inoculation with tumor cells might be involved in development of CIBP in the rat model.

Inhibition of ErbB2 signaling attenuates pain-like behavior induced in the rat model of CIBP. To clarify the role of ErbB signaling pathway in CIBP, PD168393, a selective tyrosine kinase inhibitor of ErbB2 was used in this study. The inhibitor was administered daily, intrathecally, beginning 6 days after tumor cell inoculation. As shown in Fig. 3, after 4 days of treatment with the ErbB2 inhibitor, CIBPinduced thermal hyperalgesia and mechanical allodynia were significantly attenuated 10 days after tumor cell inoculation $(\mathrm{P}<0.01$, compared to CIBP group). These protective effects were sustained until 14th day after tumor cell inoculation, afterwards gradually decreasing until day 21 , likely due to 

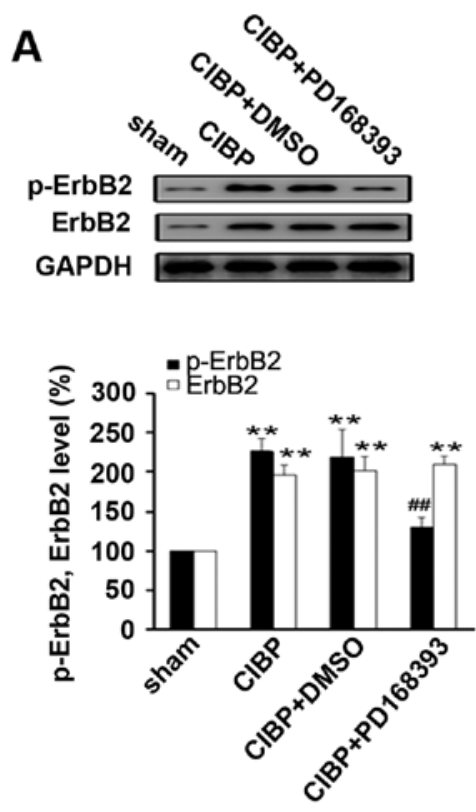

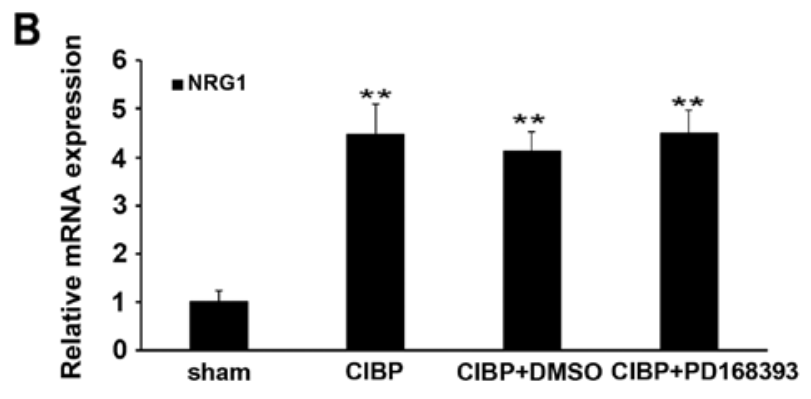

C

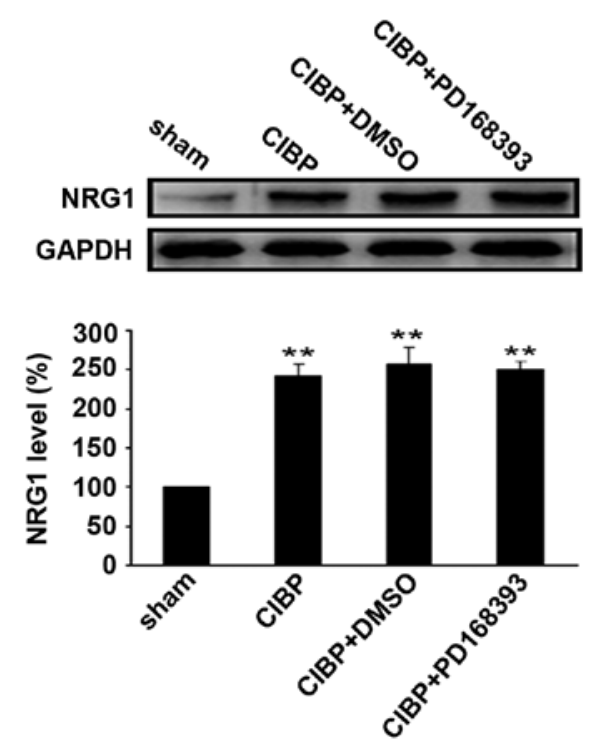

Figure 4. Effects of the ErbB2 inhibitor on the expression of ErbB2, NRG1 and level of p-ErbB2 in spinal cord tissues. (A) ErbB2 and p-ErbB2, (B) NRG1 mRNA and (C) protein in spinal cord tissues of rats were examined by q-RT-PCR and western blot analysis, respectively, after 9 days of PD168393 treatment. Relative mRNA or protein expressions (target/GAPDH) were quantified from six animal samples. ${ }^{*} \mathrm{P}<0.05,{ }^{* * *} \mathrm{P}<0.01 \mathrm{compared}$ with sham; ${ }^{* \#} \mathrm{P}<0.01$ compared with CIBP; $n=6$.

drug withdrawal at day 15 . The expression of NRG1 was not impacted by the ErbB2 inhibitor.

To confirm the inhibitory effect of PD168393 on ErbB2 signaling activity, we examined the CIBP-induced phosphorylation of ErbB2 in spinal cord tissues of rats. The results showed that the level of phosphor-ErbB2 was significantly reduced in spinal cord tissues of rats in CIBP + PD168393 group, compared with CIBP or CIBP + DMSO ( $<<0.01)$ (Fig. 4A). However, treatment with PD168393 had no effect on the expression levels of NRG1 mRNA and protein, as compared with CIBP group (Fig. 4B and C), suggesting NRG1 may act as an upstream regulator of ErbB2 signaling pathway.

Inhibition of ErbB2 signaling suppresses the activation of Akt-1 and p38MAPK induced by tumor inoculation in the rat model of CIBP. As shown in Fig. 5A and $\mathrm{B}$, activation of Akt-1 and P38MAPK was detected in rats with intratibia injection of tumor cells. The phosphorylation level of Akt-1 and P38MAPK gradually increased from the 6th day after tumor inoculation, and reached a peak at the 14th day, then decreased until day 21. After 9 days of treatment with PD168393, the mRNA expressions of Akt-1 and P38MAPK were greatly reduced and the protein levels of p-Akt-1 and p-P38MAPK were significantly reduced in spinal cord tissues of rats, as compared with those in CIBP group $(\mathrm{P}<0.01)$ (Fig. 5C-E). However, no significant difference in levels of p-Akt-1 and p-P38MAPK was found between CIBP group and CIBP + DMSO group (Fig. 5D and E).

The administration of exogenous NRG1 provokes pain-like behavior in the rat model of CIBP. To validate the effect of NRG1 on pain, we observed the pain-like behavior of rats with intrathecal injection of exogenous NRG1. We found that intrathecal administration of exogenous NRG1 (HRG1 $\beta$ ) could trigger thermal hyperalgesia and mechanical allodynia in rats immediately after injection. In our observation, the values of PWL and PWT were significantly reduced after an administration of HRG1 $\beta$ in a time-dependent pattern $(\mathrm{P}<0.01$, compared with sham group) (Fig. 6). However, co-treatment with HRG1 $\beta$ and PD168393 relieved the exogenous NRG1induced thermal hyperalgesia and mechanical allodynia ( $\mathrm{P}<0.01$ compared to treatment with HRG1 $\beta$ alone) (Fig. 6).

The administration of exogenous NRG1 induces the activation of ErbB2, Akt-1 and p38MAPK, which could be blocked by ErbB2 inhibitor. Our study also found that administration of exogenous NRG1 induced an increase in mRNA and protein expression of ErbB2, as well as the level of p-ErbB2 
A
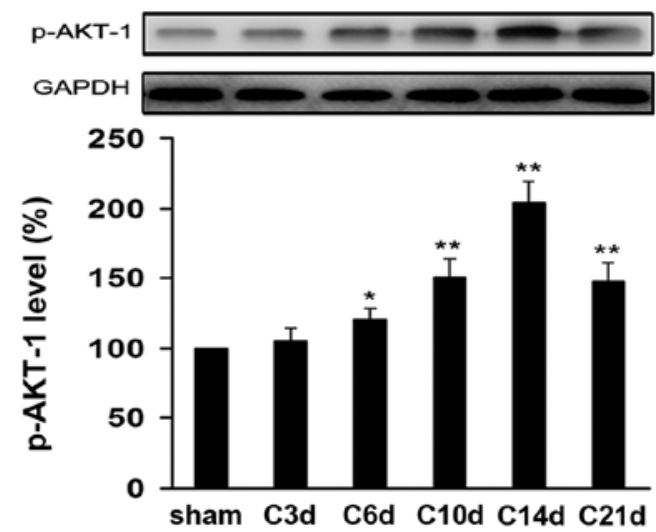

B

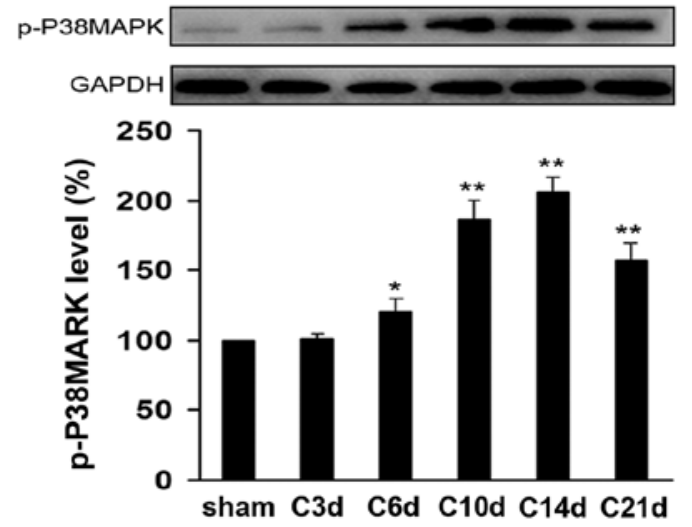

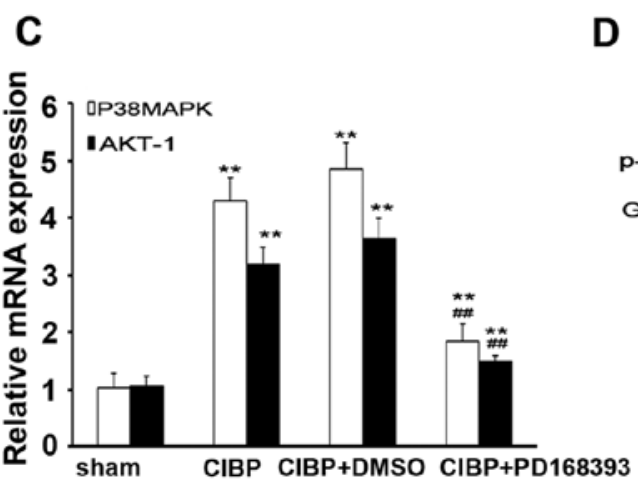
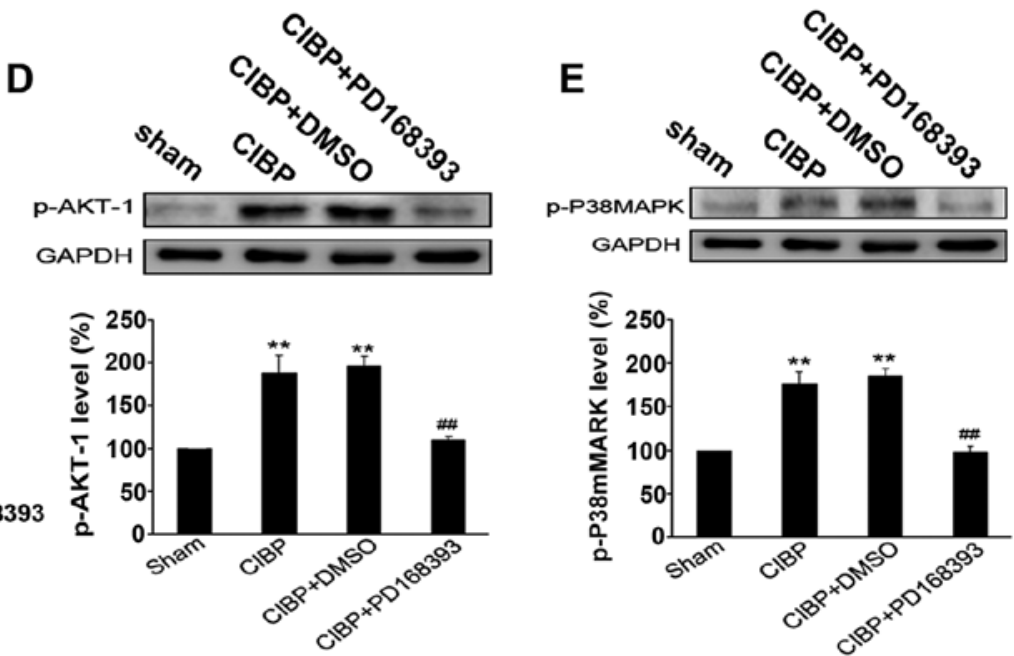

Figure 5. Effects of the ErbB2 inhibitor on the levels of p-Akt-1 and p-P38MAPK in spinal cord tissues. The levels of (A) p-Akt-1 and (B) p-P38MAPK in spinal cord tissues of the rat model of CIBP were examined by western blot analysis on the indicated day after Walker 256 inoculation. The expression of (C) mRNA and (D and E) protein of Akt-1 and P38MAPK in spinal cord tissues of rats between different groups assayed by q-RT-PCR and western blot analysis, respectively, after 9 days of PD168393 treatment. Relative mRNA or protein expressions (target/GAPDH) were quantified from six animal samples. ${ }^{*}<0.05$, ${ }^{* *} \mathrm{P}<0.01$ compared with sham; ${ }^{\#} \mathrm{P}<0.01$ compared with CIBP; $\mathrm{n}=6$.

A

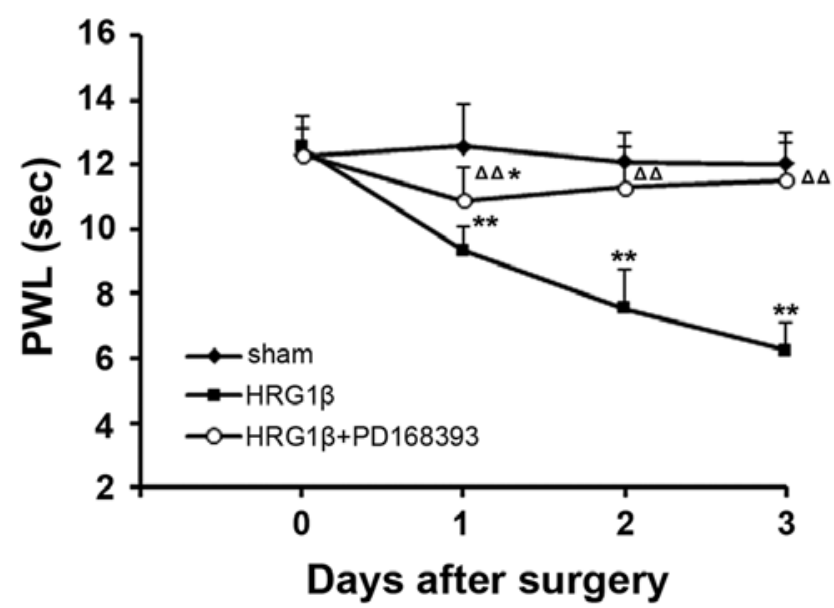

B

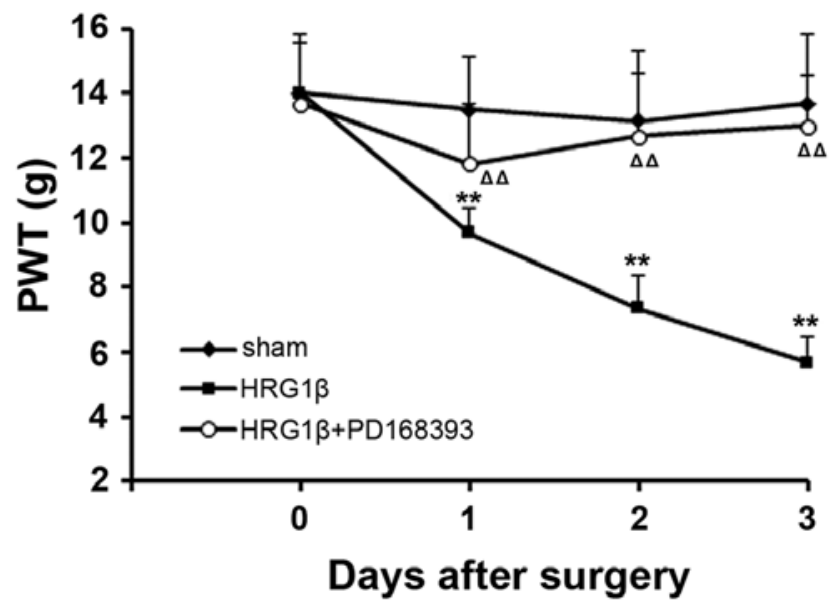

Figure 6. Administration of exogenous NRG1 provoked pain-like behavior in rats. In sham operation group, $10 \mu 1$ of normal saline was injected into the tibia cavity and $10 \mu \mathrm{l}$ of neuregulin- $\beta 1$ EGF domain (HRG1 $\beta, 0.4 \mathrm{ng} / \mu \mathrm{l})$ was injected into the tibia cavity in HRG1 $\beta$ group. In HRG1 $\beta+$ PD168393 group, the same dose of HRG1 $\beta$ was injected after 1-h treatment with $10 \mu \mathrm{g}$ PD168393. Drug injection was performed once a day for three successive days. (A) PWL and (B) PWT values were assessed on indicated day after drug injection as previously described. Data were calculated from six animals. " $\mathrm{P}<0.05{ }^{* * *} \mathrm{P}<0.01 \mathrm{compared}$ with sham; ${ }^{\triangle} \mathrm{P}<0.05,{ }^{\triangle} \mathrm{P}<0.01$ compared with HRG1 $\beta$ group; $\mathrm{n}=6$. 
A

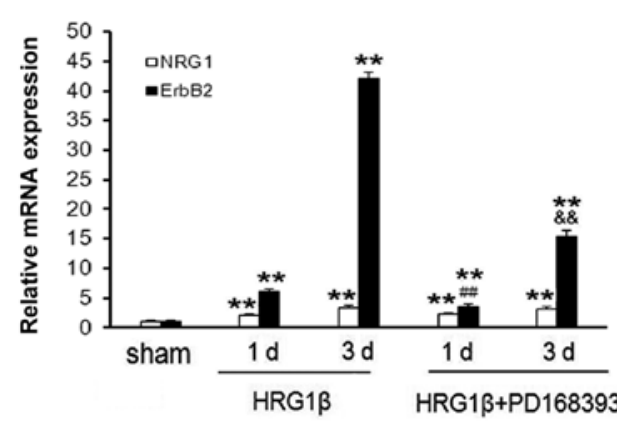

C
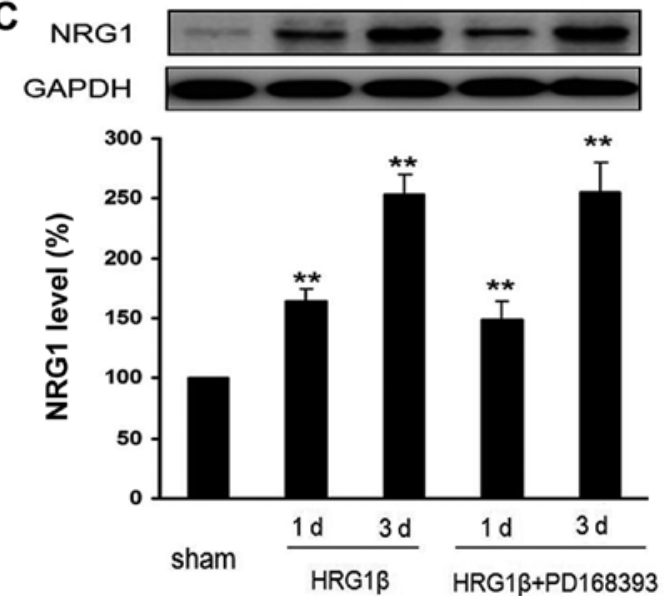

E
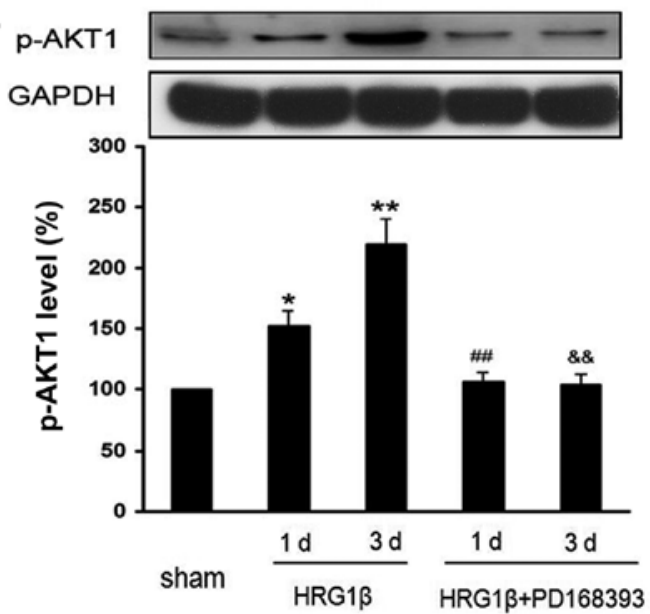

B

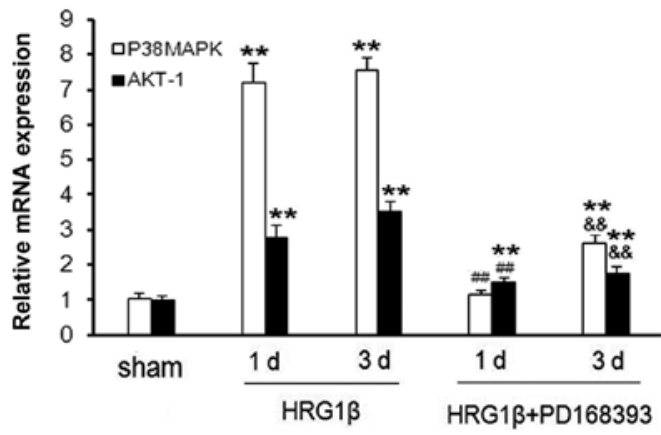

D
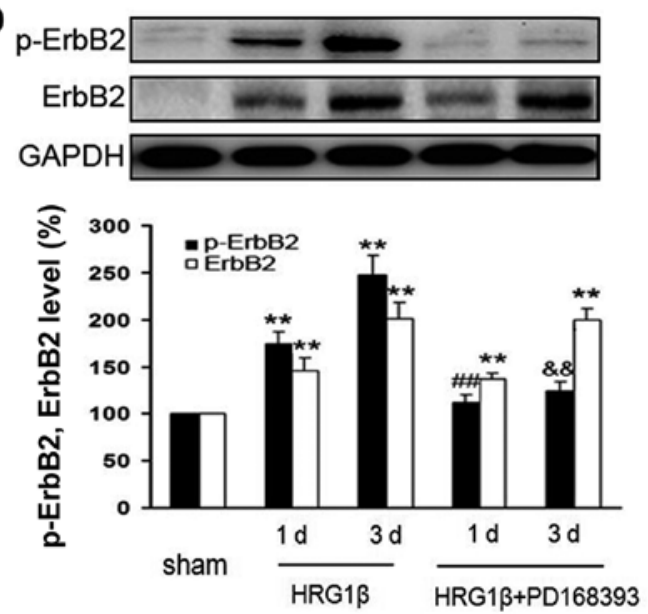

F
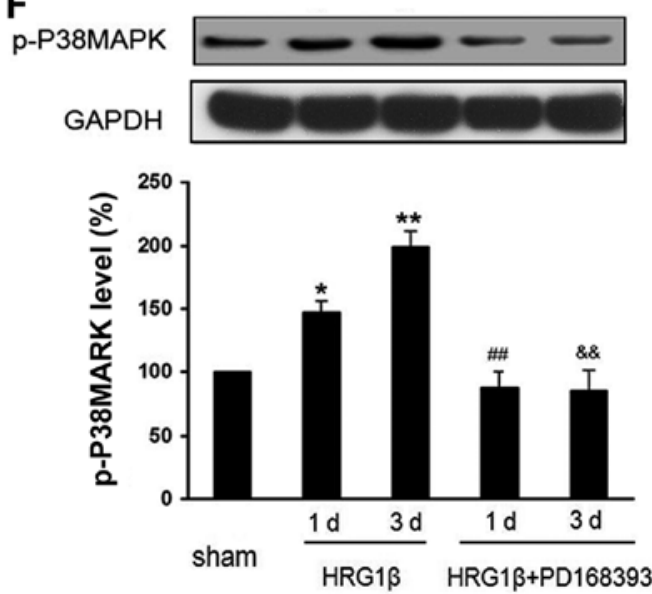

Figure 7. Administration of exogenous NRG1 induces the activation of ErbB2, Akt-1 and p38MAPK, which could be blocked by ErbB2 inhibitor. In sham operation group, $10 \mu \mathrm{l}$ of normal saline was injected into the tibia cavity and $10 \mu 1$ of neuregulin- $\beta 1$ EGF domain (HRG1 $\beta, 0.4 \mathrm{ng} / \mu 1)$ was injected into the tibia cavity in HRG1 $\beta$ group. In HRG1 $\beta$ + PD168393 group, the same dose of HRG1 $\beta$ was injected after 1-h treatment with $10 \mu \mathrm{g}$ PD168393. Drug injection was performed once a day for three successive days. (A and B) The mRNA expressions of NRG1, ErbB2, Akt-1 and p38MAPK were analyzed by q-RT-PCR. (C-F) The protein expressions were determined by western blot analysis. ${ }^{*} \mathrm{P}<0.05,{ }^{* *} \mathrm{P}<0.01$ compared with sham; ${ }^{*} \mathrm{P}<0.05,{ }^{\# \#} \mathrm{P}<0.01$ compared with HRG1 $\beta$ 1d; ${ }^{\&} \mathrm{P}<0.05$, \&\& $\mathrm{P}<0.01$ compared with HRG1 $\beta 3 \mathrm{~d} ; \mathrm{n}=6$.

(Fig. 7A, C and D). Interestingly, the activation of ErbB2 could be inhibited by co-administration of PD168393 (Fig. 7D). Nevertheless, the co-treatment with PD168393 and HRG1 $\beta$ had no impact on the expression of NRG1, as compared to the treatment with HRG1 $\beta$ alone $(\mathrm{P}>0.05)$ (Fig. 7C). Moreover, co-treatment with PD168393 and HRG1 $\beta$ also suppressed HRG1 $\beta$-induced Akt-1, p38MAPK transcription and activation (Fig. 7B, E and F), implying that ErbB2 may serve as downstream factor of NRG1 in the regulation of signaling pathways of both Akt-1 and p38MAPK.

\section{Discussion}

Emerging lines of evidence show that astrocytes and microglia in the spinal cord play essential roles in the initiation and maintenance of persistent pain induced by tissue inflammation, nerve injury and cancer (28-30). NRG1 and ErbB have been found to be expressed in the spinal cord glia $(31,32)$. The NRG1-ErbB signaling pathway has been shown to promote microglial proliferation and chemotaxis, contributing to microgliosis and pain after peripheral nerve injury (19), but its role in 
CIBP remains largely unknown. In the present investigation, we determined the involvement of NRG1-ErbB signaling using a rat CIBP model which was established by inoculation of Walker 256 tumor cells (33). Consistent with a previous report (34), bone destruction, as well as the initiation of painlike behavior occurred 6 days after tumor cell inoculation. The incidence of cortical destruction, tumor dissemination and soft tissue tumors was elevated days after tumor cell inoculation. The results of radiobioassay, histological and behavior analysis confirmed the successful establishment of a CIBP rat model.

Previous studies have shown that NRG1 activates the ErbB2 signaling pathway and promotes growth of microglial cells in the spinal cord, which may contribute to neuropathic pain following injury $(35,36)$. In accordance with those reports, we found an increase in the expression levels of NRG1 and ErbB2 in the spinal cord of rats inoculated with tumor cells. Moreover, the intrathecal administration of PD168393, an inhibitor of ErbB2 signaling, greatly reduced the CIBP-induced thermal hyperalgesia as well as mechanical allodynia the rat model of CIBP. Furthermore, LaCroix-Fralish et al reported that intrathecal administration of recombinant NRG1- $\beta 1$ protein significantly decreased the hindpaw tactile withdrawal threshold in rats (36). Consistent with their finding, our study demonstrated that intrathecal administration of exogenous NRG1 could trigger thermal hyperalgesia and mechanical allodynia through upregulation of ErbB2 and p-ErbB2 mRNA and protein levels.

Calvo et al demonstrated that in vitro, NRG1 induces activation of the ErbB2 receptor and enhances the phosphorylation of ERK1/2 and Akt, rather than the activation of p38MAPK in micoglial cells (35), however, in our study, we found the enhanced phosphorylation of both Akt-1 and p38MAPK in spinal cord tissues from the rat model of CIBP. This discrepancy may be due to the difference in animal models used between studies. Similarly, another study found an increase in phosphorylated p38 in the dorsal root ganglia (DRG) and spinal dorsal horn neurons on day 14 after an inoculation of osteosarcoma cells into the femur medullary canal (37). In that study, treatment with the p38MAPK inhibitor SCIO-469, led to a decrease in osteosarcoma-induced clinical score, with no impact on allodynia (37). Hence, the necessity of p38MAPK in CIBP needs to be further clarified. In addition, PI3K/Akt activation in the periphery has been suggested to contribute to pain behavior that was induced by capsaicin in rats (38). Intradermal administration of the PI3K inhibitor, wortmannin, dose-dependently inhibited the changes in exploratory behavior evoked by capsaicin injection (38). Here, we show that Akt signaling pathway is also activated and serves as a downstream factor of NRG1-ErbB2 signaling, as intrathecal administration of exogenous NRG1 promoted the activation of Akt-1, but inhibition of ErbB2 signaling reduced the level of p-Akt-1.

Collectively, our current study demonstrates that the NRG1-ErbB2 signaling pathway is involved in the regulation of CIBP in an experimental rat model. Akt-1 and p38MAPK kinases are also involved, and serve as downstream modulators of the NRG1-ErbB2 pathway. The roles of Akt-1 and p38MAPK in NRG1-ErbB2-mediated CIBP need to be further investigated.

\section{Acknowledgements}

This study was supported by a grant from Liaoning Science and Technology Plan Projects (no. 2012225016).

\section{References}

1. Bruera E and Kim HN: Cancer pain. JAMA 290: 2476-2479, 2003.

2. Coleman RE: Metastatic bone disease: clinical features, pathophysiology and treatment strategies. Cancer Treat Rev 27: $165-176,2001$.

3. Laird B, Colvin L and Fallon M: Management of cancer pain: basic principles and neuropathic cancer pain. Eur J Cancer 44: 1078-1082, 2008.

4. Colburn RW, DeLeo JA, Rickman AJ, Yeager MP, Kwon P and Hickey WF: Dissociation of microglial activation and neuropathic pain behaviors following peripheral nerve injury in the rat. J Neuroimmunol 79: 163-175, 1997.

5. Hains BC and Waxman SG: Activated microglia contribute to the maintenance of chronic pain after spinal cord injury. J Neurosci 26: 4308-4317, 2006.

6. Fu KY, Light AR, Matsushima GK and Maixner W: Microglial reactions after subcutaneous formalin injection into the rat hind paw. Brain Res 825: 59-67, 1999.

7. Wang XW, Li TT, Zhao J, et al: Extracellular signal-regulated kinase activation in spinal astrocytes and microglia contributes to cancer-induced bone pain in rats. Neuroscience 217: 172-181, 2012.

8. Sessle BJ: Glia: non-neural players in orofacial pain. J Orofac Pain 21: 169-170, 2007.

9. Urch C: The pathophysiology of cancer-induced bone pain: current understanding. Palliat Med 18: 267-274, 2004.

10. Wang LN, Yao M, Yang JP, et al: Cancer-induced bone pain sequentially activates the ERK/MAPK pathway in different cell types in the rat spinal cord. Mol Pain 7: 48, 2011.

11. Carraway KL III and Cantley LC: A neu acquaintance for erbB3 and erbB4: a role for receptor heterodimerization in growth signaling. Cell 78: 5-8, 1994.

12. Pentassuglia L and Sawyer DB: The role of Neuregulin-1beta/ ErbB signaling in the heart. Exp Cell Res 315: 627-637, 2009.

13. Esper RM, Pankonin MS and Loeb JA: Neuregulins: versatile growth and differentiation factors in nervous system development and human disease. Brain Res Rev 51: 161-175, 2006.

14. Hynes NE and MacDonald G: ErbB receptors and signaling pathways in cancer. Curr Opin Cell Biol 21: 177-184, 2009.

15. Huang YZ, Won S, Ali DW, et al: Regulation of neuregulin signaling by PSD-95 interacting with ErbB4 at CNS synapses. Neuron 26: 443-455, 2000.

16. Canoll PD, Musacchio JM, Hardy R, Reynolds R, Marchionni MA and Salzer JL: GGF/neuregulin is a neuronal signal that promotes the proliferation and survival and inhibits the differentiation of oligodendrocyte progenitors. Neuron 17: 229-243, 1996.

17. Rio C, Rieff HI, Qi P, Khurana TS and Corfas G: Neuregulin and erbB receptors play a critical role in neuronal migration. Neuron 19: 39-50, 1997.

18. Mei L and Xiong WC: Neuregulin 1 in neural development, synaptic plasticity and schizophrenia. Nat Rev Neurosci 9: 437-452, 2008.

19. Calvo M, Zhu N, Tsantoulas C, et al: Neuregulin-ErbB signaling promotes microglial proliferation and chemotaxis contributing to microgliosis and pain after peripheral nerve injury. J Neurosci 30: 5437-5450, 2010.

20. Medhurst SJ, Walker K, Bowes M, et al: A rat model of bone cancer pain. Pain 96: 129-140, 2002.

21. Zhang YQ, Ji GC, Wu GC and Zhao ZQ: Excitatory amino acid receptor antagonists and electroacupuncture synergetically inhibit carrageenan-induced behavioral hyperalgesia and spinal fos expression in rats. Pain 99: 525-535, 2002.

22. Fry DW, Bridges AJ, Denny WA, et al: Specific, irreversible inactivation of the epidermal growth factor receptor and erbB2, by a new class of tyrosine kinase inhibitor. Proc Natl Acad Sci USA 95: 12022-12027, 1998.

23. Song XJ, Wang ZB, Gan Q and Walters ET: cAMP and cGMP contribute to sensory neuron hyperexcitability and hyperalgesia in rats with dorsal root ganglia compression. J Neurophysiol 95: 479-492, 2006. 
24. Wang ZB, Gan Q, Rupert RL, Zeng YM and Song XJ: Thiamine, pyridoxine, cyanocobalamin and their combination inhibit thermal, but not mechanical hyperalgesia in rats with primary sensory neuron injury. Pain 114: 266-277, 2005.

25. Tal M and Bennett GJ: Extra-territorial pain in rats with a peripheral mononeuropathy: mechano-hyperalgesia and mechano-allodynia in the territory of an uninjured nerve. Pain 57: 375-382, 1994.

26. Schwei MJ, Honore P, Rogers SD, et al: Neurochemical and cellular reorganization of the spinal cord in a murine model of bone cancer pain. J Neurosci 19: 10886-10897, 1999.

27. Livak KJ and Schmittgen TD: Analysis of relative gene expression data using real-time quantitative PCR and the 2(-Delta Delta C(T)) method. Methods 25: 402-408, 2001.

28. Ye J, Wang T, Han LS, et al: Diagnosis, treatment, follow-up and gene mutation analysis in four Chinese children with biotinidase deficiency. J Inherit Metab Dis 32: S295-S302, 2009.

29. Inoue K and Tsuda M: Microglia and neuropathic pain. Glia 57: 1469-1479, 2009.

30. Hald A, Nedergaard S, Hansen RR, Ding M and Heegaard AM: Differential activation of spinal cord glial cells in murine models of neuropathic and cancer pain. Eur J Pain 13: 138-145, 2009.

31. Gerecke KM, Wyss JM, Karavanova I, Buonanno A and Carroll SL: ErbB transmembrane tyrosine kinase receptors are differentially expressed throughout the adult rat central nervous system. J Comp Neurol 433: 86-100, 2001.
32. Dimayuga FO, Ding Q, Keller JN, Marchionni MA, Seroogy KB and Bruce-Keller AJ: The neuregulin GGF2 attenuates free radical release from activated microglial cells. J Neuroimmunol 136: 67-74, 2003.

33. Simpkins H, Lehman JM, Mazurkiewicz JE and Davis BH: A morphological and phenotypic analysis of Walker 256 cells. Cancer Res 51: 1334-1338, 1991.

34. Mao-Ying QL, Zhao J, Dong ZQ, et al: A rat model of bone cancer pain induced by intra-tibia inoculation of Walker 256 mammary gland carcinoma cells. Biochem Biophys Res Commun 345: 1292-1298, 2006.

35. Calvo M, Zhu N, Grist J, Ma Z, Loeb JA and Bennett DL: Following nerve injury neuregulin-1 drives microglial proliferation and neuropathic pain via the MEK/ERK pathway. Glia 59: 554-568, 2011 .

36. Lacroix-Fralish ML, Tawfik VL, Nutile-McMenemy $\mathrm{N}$ and Deleo JA: Neuregulin 1 is a pronociceptive cytokine that is regulated by progesterone in the spinal cord: implications for sex specific pain modulation. Eur J Pain 12: 94-103, 2008.

37. Svensson CI, Medicherla S, Malkmus S, et al: Role of p38 mitogen activated protein kinase in a model of osteosarcomainduced pain. Pharmacol Biochem Behav 90: 664-675, 2008.

38. Sun R, Yan J and Willis WD: Activation of protein kinase B/Akt in the periphery contributes to pain behavior induced by capsaicin in rats. Neuroscience 144: 286-294, 2007. 